\title{
CORRIGENDA
}

\section{Some Water-soluble Vitamins in the Sweat of Tropically Acclimatized European Men}

By J. W. H. LUGG AND F. P. ELLIS

Vol. 8 (I954), no. I

P. 75, Table I, heading to ninth column:

For Thiamine (mg/100 ml.) read Thiamine ( $\mu \mathrm{g} / \mathrm{I} 00 \mathrm{ml}$.$) .$

P. 76 , lines 41 and 42 :

For (range $0.095^{-0.1} 40 \mathrm{mg} / 100 \mathrm{ml}$.; mean $0.123 \mathrm{mg} / \mathrm{I} 00 \mathrm{ml}$.)

read (range $0.095^{-0} \cdot 140 \mu \mathrm{g} / 100 \mathrm{ml}$; mean $0.123 \mu \mathrm{g} / \mathrm{I} 00 \mathrm{ml}$.)

P. 77, Summary, para. I, line 4 :

For $0.123 \mathrm{mg}$ thiamine/100 $\mathrm{ml}$. read $0.123 \mu \mathrm{g}$ thiamine/100 $\mathrm{ml}$. 